\title{
The Human Face Detection Algorithms Research and exploration Based on Adaboost
}

\author{
Yinyan Li, Xueqin Lu* \\ Ningbo DaHongYing University, Ningbo, 315175, china, E-mail: lyy401@tom.com
}

\begin{abstract}
Keywords: Human Face Detection, detector, AdaBoost
Abstract: In this thesis, we implement a fast human face detection system based on the the latest real-time feature detection algorithms presented by Paul Viola [1]. Based on the implementation of the algorithms developed by Paul, we developed a quick image scanning algorithm and an algorithm for simple classifier initialization and its optimization and a method for image data preprocess of the training system. Through the experiment, we try to get the optimal parameters to construct a human face detector with high detection speed and detection rate. Finally, we will have an analysis on our experiment results and on the issue of feature selection with different system parameters and the detection result of the whole system.
\end{abstract}

\section{Introduction}

In the biology trick recognition technology, the human face detection technology developed rapidly. Relatively says, the human face detection is one kind of more direct, more convenient, more friendly, easily the non- infrigement recognition method which is accepted by the people. As the human face automatic diagnosis system first step, the human face detection technology has the extremely vital role, it provided has treated distinguishes the person face for the following person face classification the concrete information.

This thesis will firstly introduce the recent research on human face detection and compare the main methods and their limitations. In Chapter 2, we would emphasize on the feature detection algorithms. The system construction method and core algorithms will be described in Chapter 3 . Finally we will have an analysis on our experiment results.

\section{Outline of Adaboost Algorithms}

AdaBoost is one kind of the auto-adapted Boosting algorithm, it through establishes the multilearning machines combination to enable the weak learning machine the performance to obtain the promotion, because it is in sole possession of to the learning machine performance auto-adapted and to the study phenomenon immunity, in recent years has aroused the widespread interest.

The Boosting side law origin to PAC (Probably Approximately Correct, probability approximate is correct) studies the model the theoretical analysis. In [2 ] center, Kearns and Valiant proposed strong may study the concept which and be weak may study. In PAC study model, if has a multinomial study algorithm to distinguish group of concepts, and distinguishes correctly rate very high, then this group of concept is strong may study; But if the study algorithm distinguishes group of concepts correctly rate only compared to the stochastic guess slightly good, then this group of concept is weak may study; Kearns and Valiant proposed weak study algorithm and strong study algorithm equivalent question, namely whether may weakly study the algorithm to promote Cheng Qiang to study the algorithm. If two equal in value, then in study concept time, we only need to find to be allowed to promote the stochastic suspicion slightly good method it for the to be stronger than study algorithm. 
In 1990, Schapir published the first boosting method [ 3 ] Freund afterwards to make the improvement to it to propose Lu Bang method BBM (boost-by-majority) the method [ 4 ]. The Boosting method successively produces a series of learning machines in the training, each learning machine uses training collection all is from always training collection proposed a subset, each sample whether does appear in this subset is decided by before this has produced the learning machine performance, had the sample which the learning machine judges makes a mistake by a bigger probability to appear in the new training subset. This causes the learning machine which after that produces even more to concentrate to processing to had the learning machine to say the more difficult sample discrimination question. The Boosting method may strengthen assigns the algorithm to exude the ability, but also has two shortcomings: This method needs to know the weak learning machine study correct rate lower limit, but this is very difficult to achieve in the actual problem; Next, this method possibly caused the afterwards learning machine over-concentration to the minority specially difficult sample, caused the performance not to be unstable.

In 1995, Freund and Schapire proposed the Adaboost algorithm [5], is to a Boosting algorithm big enhancement.

Adaboost (Adaptive Boosting, the auto-adapted Boosting method), it no longer needs to know in advance the weak learning machine the training is wrong rate, but is comes through the dynamic adjustment various stature learning machine power value to the basic algorithm training wrong rate automatic adaption, thus has aroused the widespread interest. Adaboost training flow as follows:

Assigns: $(\mathrm{X} 1, \mathrm{Y} 1),(\mathrm{X} 2, \mathrm{Y} 2) \ldots \ldots(\mathrm{Xn}, \mathrm{Yn}), \mathrm{Xi} \in \mathrm{chi}, \mathrm{Yi} \in\{-1,+1\}$

initialization: $\mathrm{Di}(\mathrm{i})=1 / \mathrm{m}$

in th step, $(\mathrm{t}=1 \ldots \mathrm{T})$

1) acts according to distributes Dt to train the weak sorter

2) to obtain the classified result: $\mathrm{Ht}: \in-\mathrm{R}$

3) extracts wrongly rate: Epsilon $t=$ Sigma tDt (i) $\mid \mathrm{Ht}$ (i) $\square \mathrm{Yi} \mid \mathrm{AC}$

4) renews: $\mathrm{Dt}+1$ (i) $=\mathrm{Dt}$ (i) beta 1 -eit/Zt, if sample $\mathrm{Xi}$ by right distribution, then ei $=0$, otherwise ei $=1$; Beta $t=$ epsilon $t / 1$ - epsilon $t, Z t$ is turns over to a speech factor (to cause sum of the $\mathrm{Dt}+1$ is 1 , conforms to probability distribution requirement)

5) to output the final outcome:

$$
H(X)=\operatorname{sign}\left(\sum_{t=1}^{r} \alpha_{t} h_{t}(x)\right)
$$

With front boosting similar, the Adaboost method already has the sorter with the basis to always to train the centralized each sample the classified situation to adjust each sample to appear in the new training subset the probability. Different is, AdaBoost does not need to know the weak learning machine forecast accurate rate scope in advance, but is automatically acts according to the weak learning machine the forecast accurate rate hypothesis corresponding power value. Is using AdaBoost trains, the training wrong rate upper limit is a monotonous drop function, like this, so long as the basic learning machine performance can stabilize surpasses the stochastic suspicion, and 
circulates the enough long time, can wrongly cause the experience rate to fall to wilfully the low level, and has the possibility to guarantee the promotion is wrong rate also is lower than an approximate upper limit. The experimental result showed that, it to the weak learning machine stochastically measured rate auto-adapted greatly strengthened its usability. In further research, the people discovered, the boosting method although is not easy in the general case to have the study phenomenon, but to extremely sensitively classifies the noise (to be wrong to training sample mark). Because most is difficult the sample which classifies often to have the possibility is this kind of noise, but in the depending on wheel order the practical training centralism often by this kind of sample primarily, like this, this kind of noise on possibly to the always sorter usability which finally produces gets up the very tremendous influence.

\section{Based on Adaboost Method Person Face Examination Algorithm}

Paul Viola and Michael J.Jones in [ 5 ] center used one kind to obtain with the Adaboost method training based on the multi- characteristics lamination person face characteristic examination system. The person face examination algorithm leaves which with this premise compares, their system has the extremely high running rate, in has not introduced other auxiliary informations (for example between neighboring frame difference and so on) in the situation, the examination is accurate rate and wrongly acceptance rate all can achieve in the existing algorithm the best level, therefore, this algorithm has aroused the widespread interest in the person face analysis domain, this algorithm characteristic are: 1. pair of pictures do classifies, is not the direct use picture gradation value, but uses the basis which some simple picture characteristic achievement classifies. 2. this method proposed uses Adaboost to choose the minority important characteristic the method. 3. in order to further enhances the system the speed, the author also proposed a waterfall -like procedure frame, uses the Adaboost training for each until to obtain the smallest wrong rejection rate.

AdaBoost person face examination training algorithm speed very important two aspects, characteristic selection and characteristic value computation. The rectangle the characteristic vector which examines as the person face, is called the rectangular characteristic. This algorithm selected the simplest 5 rectangular characteristic template to carry on the training, by obtained as soon as applies mechanically the most suitable rectangular characteristic which Yu Renlian examined, the fact proved, this kind of characteristic selection method training speed although was not quick, but the examination efficiency was very high. Viola proposed will accumulate the detail drawing (integral image) to apply the characteristic value during the computation. Accumulates the detail drawing the quotation, may only carry on a time of calendar computation to the picture, can use the constant time to complete each characteristic value the computation, this causes the training and the examination speed big promotion.

Person Face Characteristic Choice. The person face characteristic carries on the important basis which the person face examines. With the single picture element comparison, carries on the examination with the characteristic to have very many reasons, a reason was characteristic itself has contained the partial partial information, but these partial information knowledge were very difficultly obtain through the limited training data. Uses the person face characteristic but is not the single picture element other advantage is, based on the characteristic examination must compared to based on picture element examination quick many. Therefore, the algorithm used a group to be similar to the Hall wavelet transformation filter calculates the picture the characteristic value, like Fig. (1). showed: 

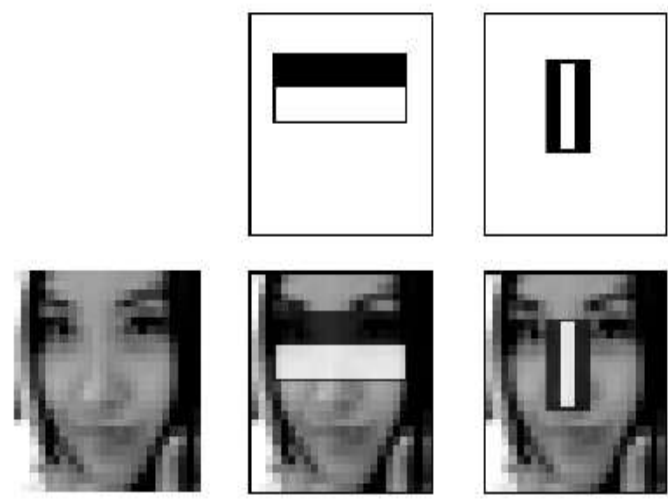

Fig. (1). On the line is the rectangular characteristic which $24 \times 24$ in the sub- window selects, gets down the line is the sub- window examines and the rectangular characteristic match.

Face some characteristics can simply describe by the rectangular characteristic, for example, usually, the eye must be deeper than the cheeks color; The bridge of the nose two sides must have to be deeper than the bridge of the nose color; The mouth must be deeper than the periphery color. Regarding a $24 \times 24$ detector, the among them rectangular characteristic quantity surpasses 1.60 , million, must select the appropriate rectangular characteristic through the specific algorithm, and can examine its group of syntheses strong sorter the person face.

We will use the simple rectangular combination to take our characteristic template. Rectangular characteristic to some simple graphic constructions, for instance edge, line segment, quite sensitive, but it only can describe the specific trend (horizontal, vertical, opposite angle) structure, therefore quite is sketchy. This kind of characteristic template all is entire and so on the rectangular neighboring combination becomes by two or many, in the characteristic template has the white and the black two kind of rectangles (defines on left angle is a white, then interlocks in turn), and defines this characteristic template characteristic value for the white rectangle picture element and subtracts the black rectangle picture element sum. Simplest 5 characteristics template: Like Fig. (2).

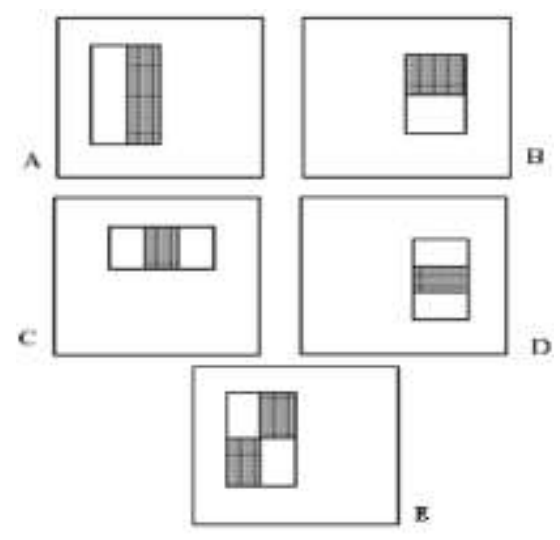

Fig. (2). Characteristic template chart

In Fig. (2). including 3 kind of characteristics: Double rectangular characteristic (two-rectangle feature), three rectangular characteristics (three-rectangle feature) and four rectangular characteristics (four-rectangle feature). Separately corresponds in the chart A, B, C, D and E. Pair of rectangular characteristic defines as the chart in the neighboring two rectangles sum of the picture element color difference. Three rectangular characteristics definition is two side two rectangles subtracts in the middle rectangle the picture element sum of color value. Four rectangular characteristics define as opposite angle on-line in the rectangle sum of the picture element color difference. 
Human Face Characteristic Computation. In order to fast calculates the picture under the different criterion the characteristic, the algorithm has introduced accumulates the detail drawing (integeralimage) the concept, like Fig. (3) shows.

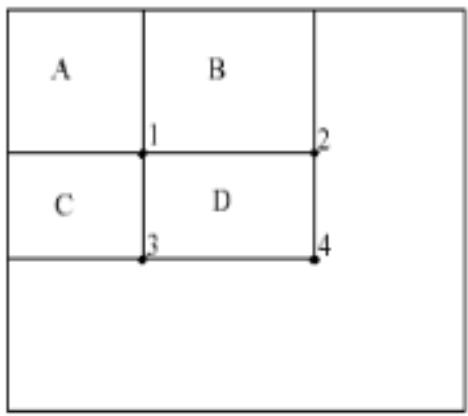

Fig. (3). Integral picture

Accumulates in the detail drawing each spot $(\mathrm{x}, \mathrm{y})$ value for picture in $(\mathrm{x}, \mathrm{y})$ on left partial all Picture element points accumulation:

$$
i i(x, y)=\sum_{x^{\prime} \leq x, y^{\prime} \leq y} i\left(x^{\prime}, y^{\prime}\right)
$$

Once calculates the product detail drawing which the picture corresponds, can calculate in the free criterion picture characteristic in the constant time. Like the chart shows, in 1, 2, 3, 4 products detail drawings value respectively is $\mathrm{A}, \mathrm{A}+\mathrm{B}, \mathrm{A}+\mathrm{C}, \mathrm{A}+\mathrm{B}+\mathrm{C}+\mathrm{D}$, but the $\mathrm{D}$ rectangular characteristic may through $4+1-(2+3)$ calculate, induces has under the following formula computation free criterion the picture characteristic:

Once calculates the product detail drawing which the picture corresponds, can calculate in the free criterion picture characteristic in the constant time. Like the chart shows, in 1, 2, 3, 4 products detail drawings value respectively is $\mathrm{A}, \mathrm{A}+\mathrm{B}, \mathrm{A}+\mathrm{C}, \mathrm{A}+\mathrm{B}+\mathrm{C}+\mathrm{D}$, but the $\mathrm{D}$ rectangular characteristic may through $4+1-(2+3)$ calculate, induces has under the following formula computation free criterion the picture characteristic:

$$
\begin{aligned}
& S(x, y)=s(x, y-1)+i(x, y) \\
& \text { ii }(x, y)=i i(x-1, y)+s(x, y)
\end{aligned}
$$

ii $(\mathrm{x}, \mathrm{y})$ for accumulates in the detail drawing $(\mathrm{x}, \mathrm{y})$ a value, $\mathrm{s}(\mathrm{x}, \mathrm{y})$ is in the picture $(\mathrm{x}, \mathrm{y})$ in the below following vector sum of color value. I $(x, y)$ is $(x, y)$ picture element value.[7] Thus, so long as to inputs the picture to spread calendar, may obtain accumulates the detail drawing, may obtain under the free criterion in the constant time the picture characteristic.

Classified function study. The algorithm supposition so long as can construct a better sorter with the minority some characteristics. In order to achieve this point, the algorithm slightly makes the improvement to the Adaboost method, stipulated: Each weak learning machine only permits based on a characteristic, like this while screened the weak learning machine also to complete in fact to the characteristic choice. In the training each in step, carries on the test to each kind of characteristic classified result. Selects wrong rate lowest to join to the final system in.

The algorithm most can differentiate the sample and the negative sample rectangular characteristic through the choice. To each characteristic, the weak sorter decides a most superior 
classified function the threshold value, causes the least samples by the mis-classification. A weak sorter (hj (x)) therefore contains characteristic fj, threshold value theta $\mathrm{j}$ and uses in to control the inequality direction $\mathrm{pj}$ :

$$
h_{j}(x)=\left\{\begin{array}{l}
1, \text { ifp }_{j} f_{j}(x)<p_{j} \theta_{j} \\
0, \text { otherwise }
\end{array}\right.
$$

$\mathrm{X}$ is in the picture $24 \times 24$ sub- window. Like this obtains is the single weak sorter, in order to obtain a confidence level higher strong sorter, the algorithm will use Adaboost many weak sorters combination to produce a strong sorter, specifically studied the algorithm will be:

Supposes $(\mathrm{X} 1, \mathrm{Y} 1) \ldots(\mathrm{Xn}, \mathrm{Yn})$ is the input picture, $\mathrm{Yi}=0,1$ uses for to mark is the negative sample or the sample.

1. initialization power value $\mathrm{w} 1, \mathrm{I}=1 / 2 \mathrm{~m}, 1 / 21$ separately corresponds the sample $(\mathrm{Yi}=1)$ and the negative sample $(\mathrm{Yi}=0)$

2. for $\mathrm{t}=1 \ldots \mathrm{T}$

the power value standardization, causes Wt is totally leads the distribution.

$$
w_{t, i}<-\frac{w_{t, i}}{\sum_{j-1}^{n} w_{t, j}}
$$

to each characteristic $\mathrm{j}$, trains sorter hj, causes this sorter only to use this characteristic to carry on the classification. The corresponding error has the smallest classified error for

$$
\varepsilon_{j}=\sum_{t} w_{i}\left|h_{j}\left(x_{i}\right)-y_{i}\right|
$$

the choice the sorter ht renewal power value,

$$
w_{t+1, i}=w_{t, i} \beta_{t}^{1-e i}
$$

if $\mathrm{Xi}$ by correct classification, ei=0, otherwise $\mathrm{ei}=1, \quad \beta=\frac{1-e_{i}}{t}$

the final strong sorter is:

$$
h(x)=\left\{\begin{array}{l}
1, \sum_{t=1}^{T} \alpha_{t} h_{t}(x) \geq \frac{1}{2} \sum_{t=1}^{T} \alpha_{t} \alpha_{t}=\log \frac{1}{\boldsymbol{\beta}_{t}} \\
0, \text { otherwise }
\end{array}\right.
$$


The chart which points out in front of this article 1 first behavior characteristic filter hints, the second line as soon as lists as by the mapping, the latter two rows respectively be put the filter on the model target sector situation. Looked from the experimental result that, the detector obviously separately used the eye region brightness to be lower than the cheeks and binocular brightness is lower than the glabella the characteristic.

Based on Adaboost Waterfall Type Person Face Examination Algorithm. In order to further enhances the system the speed, the algorithm also proposed a waterfall -like procedure frame, uses the Adaboost training for each until to obtain the smallest wrong rejection rate, if the wrong rejection rate is higher than the goal, then introduces the new level. Each when training all only aims at possesses carries on the training through its upper formation sorter sample. In this the method goal reduces the candidate region as soon as possible, only has works as level of sorters to return to the real time only then transfer next level of more complex sorters to carry on the judgement. Structure like chart 4 shows:

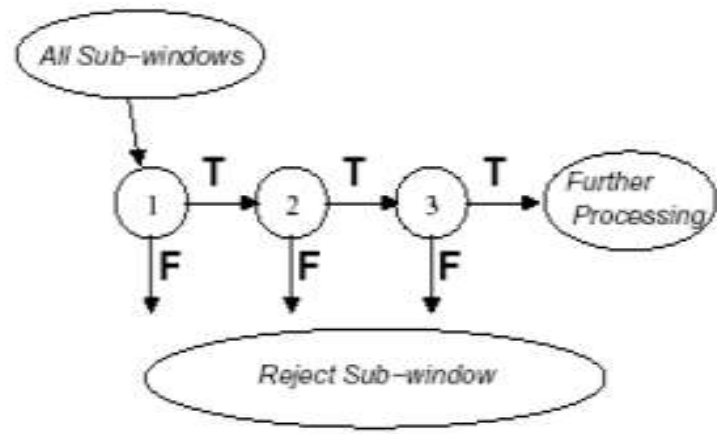

Fig. (4). The Cascade of human face Detection flow

Such procedure frame causes the more complex operation antipode minority picture element to carry on, although has used more levels (32, complete judgement needs 8,000 machine operations) but each chart only to need to carry out the average 270 operations in fact, may see, waterfall type structure big reduction system overall operand.

\section{Discussion and Conclusion}

Through the overall system design and the realization, a skeleton fast person face examination positioning system which we already succeeded. The system has not used to the colored picture in the person face skin color and the texture characteristic and so on the analysis, but is mainly based on the gradation picture in the person face characteristic in the different position gradation contrast. Such choice one stems from the examination speed consideration, two stems from the application consideration. Because the system goal is can utilize the video frequency to flow the person face examination localization, the speed naturally is most important; Moreover, many existing video frequencies gains way all is by the gradation chart way output, therefore will be able to have a more widespread application based on the gradation chart examination, also its compatibility will be more widespread, will be allowed simultaneously to apply the colored video frequency to flow the gain way.

The existing system most major problem is in its robustness. Receives the training result parameter the influence, our skeleton multilayered sorter layer too few, cannot achieve the extremely low mistake sentences to rate, this causes in the examination the output result mistake to be more, and is not good to the complex background examination effect. Creates this problem the key still to train the link. The hope can find a training collection partial characteristic extremely weak large-scale training collection picture in following work, is best can from the on-line 
stochastic selection picture and to it carries on the person eye demarcating. We also to the entire training system algorithm, will make the improvement from the speed, thus will enhance the overall system the training speed. Simultaneously finally will apply in the actual examination, and appraisal examination effect robustness. These all need long-term and the patient experimental work.

\section{Conflict of Interest}

The author confirms that this article content has no conflict of interest.

\section{References}

[1] Viola Paul, Jones Michael. Rapid Object Detection using a Boosted Cascade of Simple Features. Kauai Hawaii USAIn Proc IEEE Conf on Computer Vision and Pattern Recognition, 2004, 57(2): 137-154

[2] Ming-Hsuan Yang, Kriegman, David J, Ahuja, Narendra. Detecting Faces in Images A Survey. IEEE Transactions on Pattern Analysis \& Machine Intelligence, 2002, 24(1): 34-58

[3] Freund Y, Schapire R E. A decision-theoretic generalization of online learning and an application to boosting. Second European Conference on Computational Learning Theory, 2005, 27(2):167-173

[4] H Rowley, S Baluja, and T Kanade. Neural network-based face detection. IEEE Patt Anal Mach Intell, 1998, 20(1): 22-38

[5] Schapire R E. The Strength of weak learnability. Machine Learning, 1990, 12(5): 197-227

[6] Viola Paul, Jones, Michael J. Robust Real-Time Face Detection. International Journal of Computer Vision, 2004, 57(2): 137-154

[7] Kearns M, Valiant L G. Learning Boolean formulae for finite automata is as hardas factoring. Cambridge Harvard University Aiken Computation Laboratory, 1998, TR-1488, 21

[8] V. Nair, P. Laprise, and J. Clark, "An FPGA-based people detection system," EURASIP Journal of Applied Signal Processing, 2005(7), pp. 1047-1061, 2005

[9] C. Gao and S. Lu, "Novel FPGA based Haar classifier face detection algorithm acceleration," In Proceedings of International Conference on Field Programmable Logic and Applications, 2008.

[10] T.S. Huang and G.Z. Yang. Human Face Detection in a Complex Background, Pattern Recognition, 1994, 1

[11] B. Moghaddam and A. Pentland. Probabilistic Visual Learning for Object Recognition, IEEE Trans, Pattern Analysisand Machine Intelligence, July 1997, 19(7): 696-710.

[12] A. Samal and P.A. Iyengar, "Automatic Recognition and Analysis of Human Faces and Facial Expressions: A Survey”. Pattern Recognition, Vol. 25, No. 1, pp.65-77, 1992.

[13] J Meynet, V Popovici, J P Thiran. "Face detection with boosted Gaussian features". Pattern. Recogn., Vol. 40, pp. 2283-2291, 2007.

[14] L L Huang, A Shimizu. "Amulti-expert approach for robust face detection". Pattern Recogn., Vol. 39, pp. 1695-1703, 2006.

[15] L H Liang, H Z Ai, K Z He, et al. "Single Rotated Face Location Based on Affine Template Matching”. Chinese J. Comput., Vol. 23, No. 6, pp. 640-645, 2000.

[16] T Sergios, K Konstantinos. Pattern Recognition(Third Edition).Elsevier Inc. pp.397-425. 2006. 